\title{
The Prediction Model of Residential Housing-Price Tolerability Based on Fuzzy Matrix Analysis Theory
}

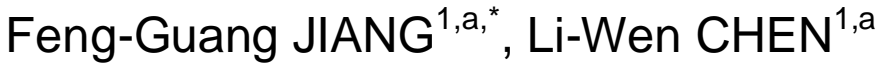 \\ ${ }^{1}$ College of Economics \& Management, Hebei University of Technology, Tianjin, 300401, China \\ aj-f-guang@163.com \\ ${ }^{*}$ Corresponding author
}

Keywords: Residential housing-price tolerability, Fuzzy matrix analysis theory, Prediction model, Housing price-income ratio.

\begin{abstract}
With the soaring property prices, research on the residential housing-price tolerability is of great practical significance to manage national economy order and control the national macro-economy policies. A prediction model of residential housing-price tolerability based on fuzzy matrix analysis theory has been put forward by this paper. Hou sing price-income ratio was used as the indicator of residential tolerability. And the principal influencing factors of housing prices have been determined. The prediction model based on fuzzy matrix has been set up according to data and realized by MATLAB program. The reasonable suggestion and conclusion on residential housing-price tolerability can be given by analyzing the results of fuzzy matrix model. The prediction model based on fuzzy matrix put forward by this paper considered several principal factors such as political, economy, administration, society and nature. The weight, which represents the importance of a certain factor, can be calculated by correlation index. The simulation result shows that the prediction model can reflect the objective reality comprehensively and effectively.
\end{abstract}

\section{Introduction}

At present, the housing price of our country is climbing up constantly. Some mainstream economists are keen on offering explanation based on superficial supply-demand relationship. It is true that the real housing price is determined by supply-demand relationship [1]. But to some extent, the causes of rising housing price can not ignore the politics, economic, administration, society and natural factors. Among all these affecting factors, residents' per capita disposable income, land prices, over 5 years loan rates and the density of population are more prominent factors [2]. The research on housing price tolerability can guide national macro-control policy and reasonable family consumption and investment $[3,4]$. The hosing problem is a big issue which related to national development strategy. To research the housing demand and variation rule of general public will be conducive to the sustainable development of urban housing construction and people's unity and social stability $[5,6]$. Housing problem is related to national economy and people's livelihood. To solve the urban residents, is one of the important part of building a harmonious socialist society.

Traditional research evaluation methods on housing prices tolerability of residents didn't give an overall consideration on the effects coming from policy, economy, administration, society and nature. The research on residential tolerability has single assessment standard which is not typical and lack of accuracy $[7,8]$. The fuzzy matrix analysis theory put forward by this paper take political, economical, social and natural factors in consideration to discuss the effect on housing price. Gathering data and analyze the affecting factors weigh by using fuzzy matrix. The fuzzy matrix, refers to making a complicated multi-objective decision making problem as a system, decompose the target into multiple goals or criterion so that to get several levels of the multi-index[9]. When we are doing research on the market development status and level, as long as the objective data and information on all aspects can be acquired, the weigh of all factors can be calculated through coefficient index and can be studied by using fuzzy matrix analysis method, which needs less information and unifies the quantitative analysis and qualitative method[10]. And this is more systematic and hierarchical with strong representation. 


\section{The fuzzy matrix analysis on housing price tolerability affecting factors}

At present, the mathematic models on housing price are based on multiple linear regression theory, nuclear density model theory and fuzzy mathematical model theory, which is more intuitive and reasonable. The fuzzy matrix analysis method is to construct criterion matrix, figure out its maximum eigen value and eigenvector $\omega$ then normalize the eigenvector, which is the importance weight of a certain level indicator against another level. Meanwhile, this thesis put forward some hypothesis based on others' relevant paper and statistic data:

(1)Assume that all data is reliable.(2)Assume that in addition to the mentioned factors in this paper, such as the political, economic, administrative, social and natural factors), the other affecting factors' impact on hou sing prices is very small and can be neglected.(3)Residential housing tolerability can be represented by housing price-income ratios.(4)International repercussions, per capita disposable income, land price, over five years loans rates have indirect effects on housing price-income ratio.

Based on these hypotheses, this thesis selected the hou sing price $\mathrm{C}$ as the parameter to be discussed, which affecting factors are international repercussions $\mathrm{C}_{1}$, per capita disposable income $\mathrm{C}_{2}$, land price $\mathrm{C}_{3}$, over five years loans rates $\mathrm{C}_{4}$, hou sing purchase restrictions policy $\mathrm{C}_{5}$, security housing policy $\mathrm{C}_{6}$, population density $\mathrm{C}_{7}$, family construction $\mathrm{C} 8$ and location $\mathrm{C}_{9}$. Paired comparison matrix is $\mathrm{A}$. The maximum eigen value is $\lambda$. The coincidence indicator is CI. The random coincidence indicator is RI. The consistency ratio is CR. The combination weight vector is $\omega$.

\section{The affecting factors of residential housing price tolerability}

This thesis use housing price index, which refers to the high-low expression form, to represent the tolerability of residents: international repercussions $\mathrm{C}_{1}$, per capita disposable income $\mathrm{C}_{2}$, land price $\mathrm{C}_{3}$, over five years loans rates $\mathrm{C}_{4}$, hou sing purchase restrictions policy $\mathrm{C}_{5}$, security hou sing policy $\mathrm{C}_{6}$, population density $\mathrm{C}_{7}$, family construction $\mathrm{C}_{8}$ and location $\mathrm{C}_{9}$. As the figure shows:

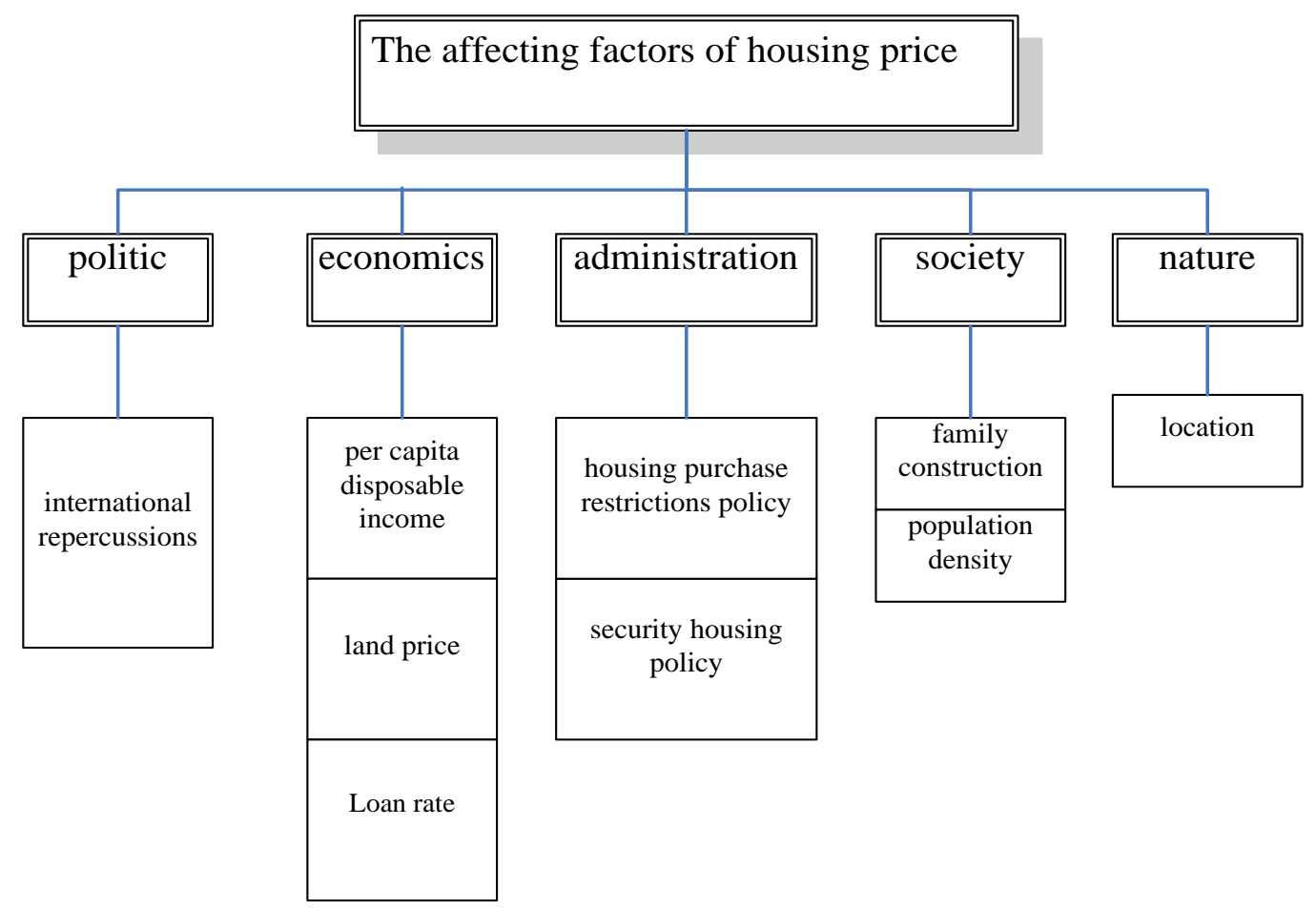

Fig. 1 The affecting factors of residential tolerability on housing price

\section{The model preparation theory}

1.The fuzzy matrix model construction method

(1)Define the discuss domain for evaluation objects: 
Factors sets: suppose there are $\mathrm{N}$ evaluation factors in discuss domain, which is evaluation index and can be expressed as: $\mathrm{U}=\{\mathrm{u} 1, \mathrm{u} 2, \ldots, \mathrm{un}\}$.

Comments sets: suppose there is $\mathrm{m}$ level in discuss domain, and can be expressed as:

$$
\mathrm{V}=\{\mathrm{v} 1, \mathrm{v} 2, . \quad . \quad, \mathrm{v} \mathrm{m}\}
$$

(2)Set up the fuzzy matrix and evaluating single factors

Making the single factor evaluation to all the factors among $\mathrm{U}$, and can get a fuzzy matrix form $\mathrm{U}$ to $\mathrm{V}$. $\mathrm{R} \in \mathrm{F}(\mathrm{U} \times \mathrm{V})$, which can be called as single factors evaluation matrix.

$$
[R]=\left[\begin{array}{cccc}
r_{11} & r_{12} & \cdots & r_{1 m} \\
r_{21} & r_{22} & \cdots & r_{2 m} \\
\cdots & \cdots & \cdots & \cdots \\
r_{n 1} & r_{n 2} & \cdots & r_{n m}
\end{array}\right] \quad 0 \leq \mathrm{rij} \leq 1(\mathrm{i}=1,2,3, \ldots, \mathrm{n} ; \mathrm{j}=1,2,3, \ldots, \mathrm{m})
$$

According to the definition of fuzzy relationship, the element rij in [R] stands by the No.i criterion which can be seemed as the No.j geography quality belonging degree, and the specific value is given by belonging function. Once $[R] \in F(U \times V)$ is determined, and there exits a fuzzy vector $A \in F(U)$, then the only fuzzy transformation $\mathrm{B}$ from $\mathrm{U}$ to $\mathrm{V}$ can be determined.

(3)Composing the fuzzy synthesize evaluation vector

Using composing figure A combined with all evaluating object and get the goal estimated vector. The model of composed fuzzy synthesize evaluation is:

$$
B=A \circ R=\left(a_{1}, a_{2}, \cdots, a_{p}\right) \circ\left[\begin{array}{cccc}
r_{11} & r_{12} & \cdots & r_{1 m} \\
r_{21} & r_{22} & \cdots & r_{2 m} \\
\cdots & \cdots & \cdots & \cdots \\
r_{n 1} & r_{n 2} & \cdots & r_{n m}
\end{array}\right]
$$

Among this : $[\mathrm{A}]=(\mathrm{a} 1, \mathrm{a} 2, \ldots$, ap $)$ is the fuzzy sub-set of evaluation factors weight, ai is the No.i evaluation factor, which contents

$$
\sum_{i=1}^{n} a_{i}=1,[B]=(b 1, \mathrm{~b} 2, \cdots, \mathrm{bm})
$$

And the symbol "o"is the composed figure of fuzzy matrix. $\mathrm{Bj}$ is the No.j level remark of a certain evaluation object. Using the fuzzy transformation as the basic criterion, according to the principal of the largest belonging degree, the evaluating object should belong to the largest remark level of [B]. In some circumstance, to get the final belonging degree needs appropriate modulation.

(4)The vector analysis of fuzzy composed evaluation result

To use he weighting average principal method to analyze the fuzzy composed evaluation vector. Regard the level as a related position and make it continuous. In order to deal it quantitatively, using " 1,2, ,m" to represent each level and named as rank. To sum all level rank in B and get the related position of evaluation object. This can be expressed as:

$$
Q=\sum_{j=1}^{m}\left(b_{j}^{k} \cdot j\right) / \sum_{j=1}^{m}\left(b_{j}^{k}\right)
$$

The fuzzy mathematics can rank the effects of housing price tolerability, per capita disposable income, over five year loan rate, population density, housing price index and family construction.

2. The analysis of data

By the data from internet and gathering the housing price index, per capita disposable income and land price, over five year loan rate, using SPSS to realize the relevant analysis. 
Table 1 Correlations

\begin{tabular}{|c|c|c|c|c|c|}
\hline & & Housing price index & Income & Loan rate & Land price \\
\hline \multirow{3}{*}{ Housing price index } & Pearson Correlation & 1 & -.105 & .163 & -.161 \\
\hline & Sig. (2-tailed) & & .805 & .699 & .703 \\
\hline & $\mathrm{N}$ & 8 & 8 & 8 & 8 \\
\hline \multirow{4}{*}{ Income } & Pearson Correlation & -.105 & 1 & .273 & $.973^{* *}$ \\
\hline & Sig. (2-tailed) & .805 & & .513 & .000 \\
\hline & $\mathrm{N}$ & 8 & 8 & 8 & 8 \\
\hline & Pearson Correlation & .163 & .273 & 1 & .119 \\
\hline \multirow[t]{3}{*}{ Loan rate } & Sig. (2-tailed) & 699 & .513 & & .779 \\
\hline & $\mathrm{N}$ & 8 & 8 & 8 & 8 \\
\hline & Pearson Correlation & -.161 & $.973^{* *}$ & .119 & 1 \\
\hline \multirow[t]{2}{*}{ Land price } & Sig. (2-tailed) & .703 & .000 & .779 & \\
\hline & $\mathrm{N}$ & 8 & 8 & 8 & 8 \\
\hline
\end{tabular}

From the table, we can get that:

$\operatorname{Corr}(\mathrm{C}, \mathrm{C} 2)=0.805 ; \operatorname{Corr}(\mathrm{C}, \mathrm{C} 3)=0.703 ; \operatorname{Corr}(\mathrm{C}, \mathrm{C} 4)=0.699$

The correlation coefficients refers to the relevant degree between two variables, $\operatorname{Corr}(\mathrm{X}, \mathrm{Y})>0$ stands for positive relevance, and $\operatorname{Corr}(\mathrm{X}, \mathrm{Y})<0$ stands for negative relevance. The statistics shows that housing price index, per capita disposable income and land price, over five year loan rate are positive relevance. According to the coefficient factor we can get the importance degree among $\mathrm{C} 2$, C3, C4. First of all, the measure scale is defined as 1-9, because the psychologist considered the scale is accord with people's management scale, it's simple and convenient with reasonable result. We can draw two conclusion and making reasonable hypothesis:

(1) C2, C3, C4 has the same importance degree for housing price index, and suppose $\mathrm{a} 23=\mathrm{a} 24=\mathrm{a} 34=1$;

(2) $\mathrm{C} 2, \mathrm{C} 3, \mathrm{C} 4$ has large impact on housing price, suppose the ratio for comparing them with the other secondary factor is 8 .

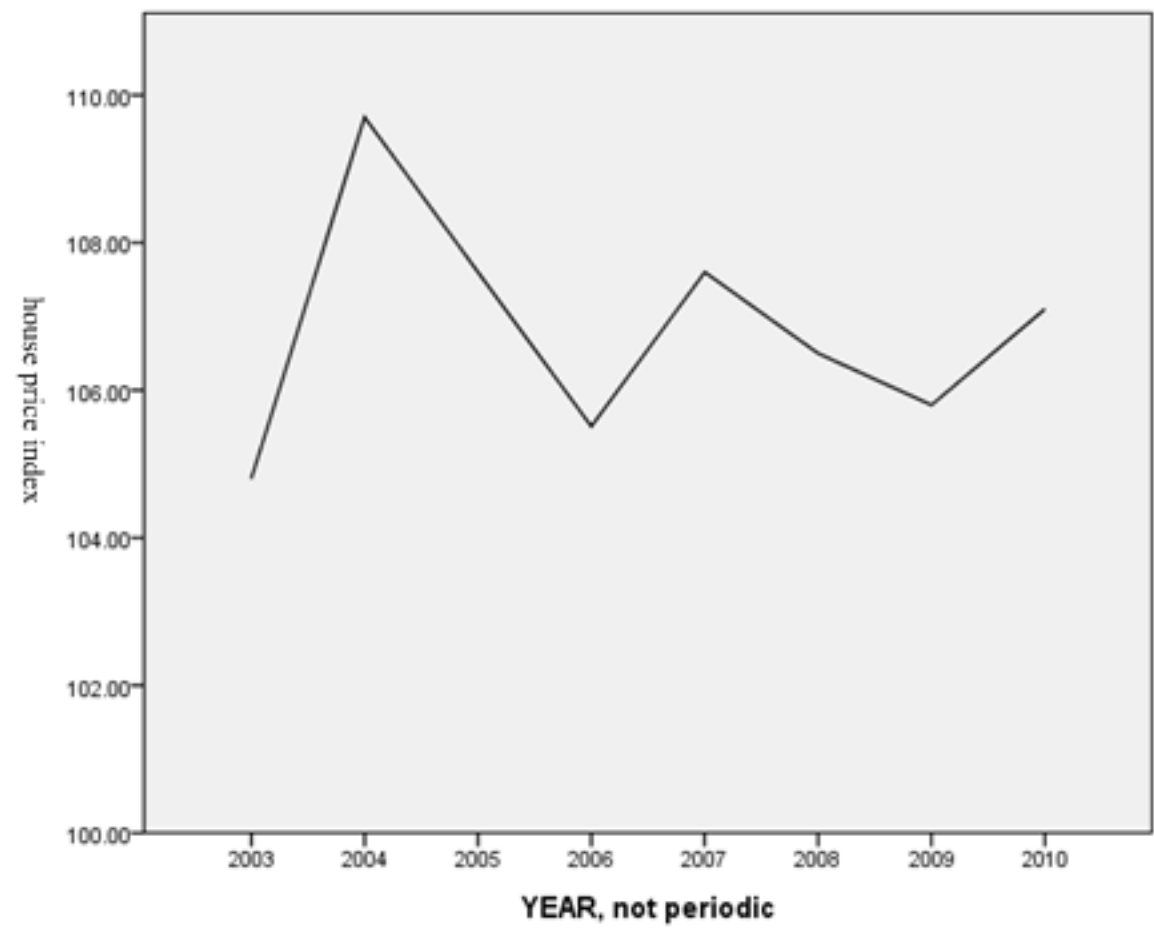

Fig. 2 The housing price index 


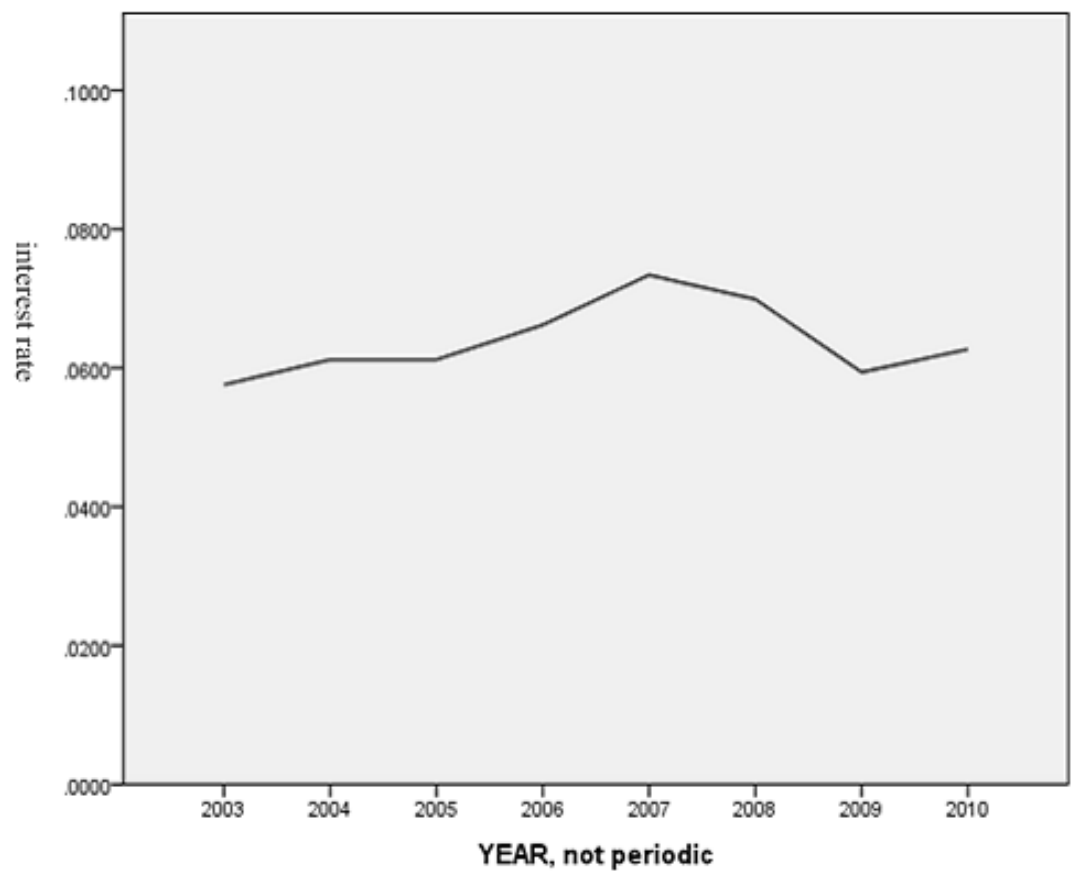

Fig. 3 The loan rate

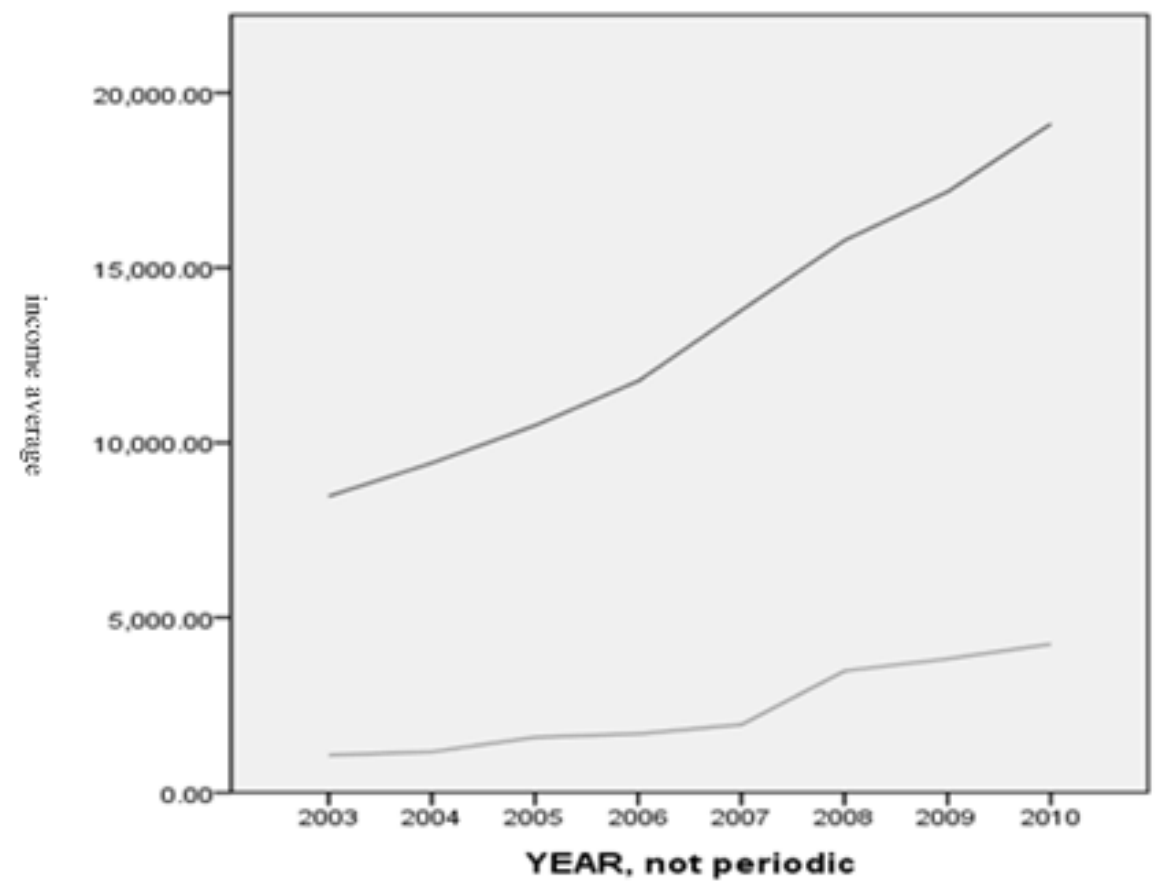

Fig. 4 The per capita disposable income average

\section{Construction of the mathematic model based on statistics}

\section{The gathered datas}

(1)The Table 2-4 below is the housing price and residential per capita pure income data form 2003 to 2012 published by state authorities. 
Table 2 The urban residential land price from 2003-2010 (unit: yuan/square meters)

\begin{tabular}{lc}
\hline Year & Residential land price \\
\hline 2003 & 1070 \\
2004 & 1166 \\
2005 & 1582 \\
2006 & 1681 \\
2007 & 1941 \\
2008 & 3479 \\
2009 & 3824 \\
2010 & 4245 \\
\hline
\end{tabular}

\begin{tabular}{|c|c|c|c|c|c|c|c|c|c|c|}
\hline Item & 2003 & 2004 & 2005 & 2006 & 2007 & 2008 & 2009 & 2010 & 2011 & 2012 \\
\hline Housing & 4764 & 5053 & 6788 & 8280 & 1155 & 1241 & 1379 & 2231 & 2276 & 2289 \\
\hline price & $4 / 04$ & & $0 / 88$ & $8<80$ & 3 & 8 & 9 & 0 & 7 & 7 \\
\hline Residential & 1388 & 1563 & 1765 & 1997 & 2198 & 2472 & 2673 & 2907 & 3290 & 3452 \\
\hline $\begin{array}{l}\text { per capita } \\
\text { pure income }\end{array}$ & 3 & 8 & 3 & 8 & 9 & 8 & 8 & 3 & 0 & 4 \\
\hline Bank loan & 5.76 & 6.12 & 6.12 & 6.84 & 7.83 & 5.94 & 5.94 & 6.40 & 7.05 & 6.92 \\
\hline rate & $\%$ & $\%$ & $\%$ & $\%$ & $\%$ & $\%$ & $\%$ & $\%$ & $\%$ & $\%$ \\
\hline $\begin{array}{c}\text { Housing } \\
\text { price-income } \\
\text { ratio }\end{array}$ & 13.6 & 12.8 & 15.2 & 16.4 & 21.2 & 20 & 20.8 & 30.8 & 27.6 & 29.8 \\
\hline
\end{tabular}

Table3 Annual loan rate for housing purchase and Residential per capita disposable income

\begin{tabular}{ccc}
\hline Year & Annual loan rate & income \\
\hline 2003 & $5.76 \%$ & 8472.2 \\
2004 & $6.12 \%$ & 9421.6 \\
2005 & $6.12 \%$ & 10493 \\
2006 & $6.62 \%$ & 11759.5 \\
2007 & $7.34 \%$ & 13785.8 \\
2008 & $6.99 \%$ & 15780.8 \\
2009 & $5.94 \%$ & 17174.7 \\
2010 & $6.27 \%$ & 19109 \\
\hline
\end{tabular}

\section{The construction of fuzzy matrix model}

Through consulting a large number of data, 9 important factors are found: international repercussions $\mathrm{C} 1$, per capita disposable income $\mathrm{C} 2$, land price $\mathrm{C} 3$, over five years loans rates $\mathrm{C} 4$, housing purchase restrictions policy $\mathrm{C} 5$, security housing policy $\mathrm{C} 6$, population density $\mathrm{C} 7$, family construction $\mathrm{C} 8$ and location $\mathrm{C} 9$. The housing price is $\mathrm{C}$. The fuzzy matrix model is operated under two-dimension data, however, this subject needs to deal with 7-demension data, which are Uprice-income ratio, X1i-land price, X2i-per capita disposable income, X3i-over five year loan rate, X4i-population density, X6i-family construction.

Set up the factors set and evaluation set. Evaluation factors set is:

$$
\mathrm{U}=\{\mathrm{X} 1 \mathrm{i}, \mathrm{X} 2 \mathrm{i}, \mathrm{X} 3 \mathrm{i}, \mathrm{X} 4 \mathrm{i}, \mathrm{X} 5 \mathrm{i}, \mathrm{X} 6 \mathrm{i}\}
$$

Set up rate set. Divided each single factor, say the tolerability of residents, into 4 grades. Then get the whole environment quality assessment set $\mathrm{V}=\{$ Excellent, good, medium and poor $\}$.

Through the calculation on fuzzy model and fuzzy vector of matrix, normalize the result and then get the processing result:

$\mathrm{U} 1=\{476$ (excellent),13883(good),5.76\%(medium), $0.4 \%$ (good),3.3(medium),89.5(excellent) $\}$ 
$\mathrm{U} 2=\{5053$ (excellent),15638(good),6.12\%(medium),0.7\%(good),5.3(medium),107.2(excellent) $\}$

$\mathrm{U} 3=\{6788$ (excellent),17653(good), 6.12 (medium), $-0.8 \%$ (good), 3.4 (medium), 106.3(excellent) $\}$

$\mathrm{U} 4=\{8280$ (excellent),19978(good),6.84(medium),1.2\%(good),4.2(medium),104.9(excellent) $\}$

The residential tolerability on housing price rate are:

U1 excellent, U2 excellent, U3 good, U4 good, U5 good, U6 medium, U7 medium, U8 poor,U9 poor,U10 poor.

Then constructing the comparing matrix A, using the relevant data, figure out each element through paired comparison 1-9 scale.

aii $=1(\mathrm{i}=1, \ldots, 9)$

a23, a24, a34can be figure out by correlation coefficient, and a23=a24=a34=1. $\quad a_{i j}=1 / a_{j i}$

The rest element can be decided by historical experiences and subjective judgment. The construction process guided by rules that: if aik $>1$, aki $>1$, then make sure aij $>1$. Because aik $>1$, aki $>1$ means $\mathrm{Ci}$ is more important than $\mathrm{Ck}$. And $\mathrm{Ck}$ is more important than $\mathrm{Cj}$. If aij $<1$, which means $\mathrm{Cj}$ is more important than $\mathrm{Ci}$, it is obviously is contradictory. So when construct the matrix make sure that aij>1 . By this way we can get Positive and negative contrast matrix A:

$$
A=\left(\begin{array}{ccccccccc}
1 & \frac{1}{9} & \frac{1}{9} & \frac{1}{9} & \frac{1}{2} & \frac{1}{4} & \frac{1}{6} & \frac{1}{5} & \frac{1}{2} \\
9 & 1 & 1 & 1 & 7 & 6 & 2 & 4 & 7 \\
9 & 1 & 1 & 1 & 7 & 6 & 2 & 4 & 7 \\
9 & 1 & 1 & 1 & 7 & 6 & 2 & 4 & 7 \\
2 & \frac{1}{7} & \frac{1}{7} & \frac{1}{7} & 1 & \frac{1}{2} & \frac{1}{5} & \frac{1}{4} & 1 \\
4 & \frac{1}{6} & \frac{1}{6} & \frac{1}{6} & 2 & 1 & \frac{1}{4} & \frac{1}{3} & 2 \\
6 & \frac{1}{2} & \frac{1}{2} & \frac{1}{2} & 5 & 4 & 1 & 2 & 5 \\
5 & \frac{1}{4} & \frac{1}{4} & \frac{1}{4} & 4 & 3 & \frac{1}{2} & 1 & 4 \\
2 & \frac{1}{7} & \frac{1}{7} & \frac{1}{7} & 1 & \frac{1}{2} & \frac{1}{5} & \frac{1}{4} & 1
\end{array}\right)
$$

Then there comes with consistency check.

The first step is to figure the maximum eigenvalue $\lambda$. Defined the consistency indicator is

$$
C I=\frac{\lambda-n}{n-1} \quad(\mathrm{n}=9)
$$

Introduce the random consistency indicator RI.

\begin{tabular}{llllllllllll}
\hline $\mathrm{n}$ & 1 & 2 & 3 & 4 & 5 & 6 & 7 & 8 & 9 & 10 & 11 \\
\hline $\mathrm{RI}$ & 0 & 0 & 0.58 & 0.90 & 1.12 & 1.24 & 1.32 & 1.41 & 1.45 & 1.49 & 1.51 \\
\hline
\end{tabular}

Define CR as the consistency ratio.

$C R=\frac{C I}{R I}<0.1$ which means passing the consistency test.

Normalize the eigenvector then can get the weight vector.

Considering the matrix has 9 orders, using MATLAB programming and calculating. The matrix can pass the consistency check and the weight vector is

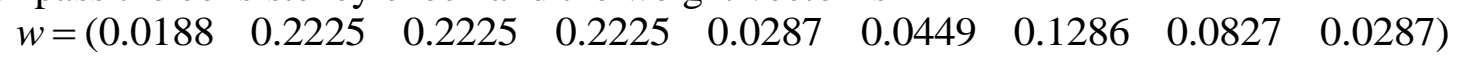

Then get the weight from $\mathrm{C} 1$ to $\mathrm{C} 9$. 


\section{The analysis based on fuzzy matrix model.}

The result of this constructed model shows that the land price dominates the housing price. So the government should balance the land price and control housing price. But meanwhile, because the density of China population is large, per capita land is limited. Although the regulation of the land price can affect the housing prices, the implementation is poor. Banks can regulate the loan rate of more than five years (because the repayment on loan is generally more than five years).There is positive correlation between loan rate and housing price. So with the guarantee of banking operation normal, the housing price can be cut down by decrease the over five year loan rate, as well as providing security housing, housing restriction, housing fund and other policies. Residential per capita income of tolerance is greater than the impact of housing price's influence on the tolerance, so the government should promote the increase on incomes with the regulations and measures, and reduce the residents living cost and tax burden so that to increase the tolerance of housing price.

\section{Reason analysis and suggestions}

\section{Moderate consumption and diversified investment}

As for a young family with housing demand, the proportion of consumer spending is high. It is recommended to improve the quality of life appropriately on meeting the basic living expenses with reduce avoidable consumer spending. The family financial plan should be done at the beginning of a year and get into the habit of budgeting and accounting. Control life expenditure within 1300 yuan per month. In addition, according to the different situation and the age, people should adjust individual investment strategy and to realize a solid strategy in different periods and ensure the effective use of different situations.

\section{Long-term investment and value-added assets}

Financial management is a way of life. Do not think that money was useless. Penny also can be turned into a lot of money with perseverance. The sooner you start saving investment, the easier it is to accumulate wealth for family. It is recommended to develop regular forced investment with 1000 yuan a month. To prepare children education foundation and old-aged pensions as soon as possible. Considering the purchase plan, 70000 yuan can deposit recycled do short or medium bank financial products.

\section{Strengthening the government supervision}

Standardize the construction of housing security system scientifically and satisfy the reasonable housing needs of low-income urban residents. With climbing housing price, effective measures should be taken at once. Focus on solving the housing problems of low-income families. Meanwhile, accelerate the construction of low-rent housing, develop the secondary housing market and rental market. The government should accelerate the construction of housing rental market standardization and legalization. Through a variety of empty houses, make full use of the existing housing resources. Only in this way can effectively solve the problem which is low and middle-income residents housing difficulty.

\section{Complete the tax policy and control speculative demand reasonable rationally.}

Formulate the tax policy of Housing transfer link. Suppress the speculative demand rationally. The reasonable housing consumption demand can maintain and promote the sustained and healthy development of real estate industry and national economic power, while maintaining a modest speculative demand on the market. However, the excessive speculative demand in the market will influence the market operation order, so the government must take measures to regulate this kind of speculative demand, and increase the restriction on land holding tax collection. 


\section{Conclusions}

In summary, residents' tolerability for housing prices is becoming gradually weak. Because housing is not only belong to the scope of economics, but also closely related to social and political. The increase of housing price can not only create wealth for society but to make the social wealth flow to the high income class from the low income class, so that to enlarge the income gap of urban residents, which can affect the construction of harmonic society. Facing the decreasing residential price tolerability, and making the estate policy scientifically is of great significance. The study on residential housing price tolerability based on fuzzy matrix analysis can compare and measure comprehensively. Take the housing - price tolerability ratio into consideration has high efficiency, and the tolerability can be used as the index of monitoring macro-economic operation with strong representation, which can offer dramatic criterions for formulation of estate industry policy scientifically.

\section{Acknowledgement}

This research was financially supported by the university natural science youth fund projects of Hebei province.

\section{References}

[1] Zheng Yao, Huaping Sun, Changchun Feng, The analysis of the influence factors of urban housing price change-based on the research data of 31 cities[J];The economic construction;2011 (007).

[2] Longtao, Li Zhangning, Bai Wanyun, Han Datao; Multiple regression analysis of house-price index factors of;[J]; Market Forum;2011 (007).

[3] Ma Jingyi; The analysis of the influencing factors and countermeasures of high prices;[J];The modern enterprise;2011 (001).

[4] Jia Shenghua. The study about the theory of the commodity residential house price influence factors and quantitative analysis, Beijing: Renmin university of China, 2006.

[5] Jiang Qiyuan, Xie Jinxing, Ye Jun; Mathematical modeling (The third edition);[M].Beijing: higher education press, 2003.8.

[6] Ou Yanhao. Model to predict the real estate price index of Changchun based on ARMA: Northeast University of finance, 2003.

[7] Minfeng Lu. Reasonable control prices level of the law of value-based on analysis of the tolerance of urban housing prices.

[8] Fengguang Jiang. The housing prices tolerance research report of Tianjin urban residents. [D]: $\mathrm{PhD}$ students academic report of Hebei university of technology.

[9] Data Source: national bureau of statistics web site.

[10]Data Source: Tianjin statistical yearbook. 